\title{
Evaluación bibliométrica de la investigación formativa en la Facultad de Medicina Veterinaria y Zootecnia de la Universidad Peruana Cayetano Heredia en el periodo 2012-2017
}

Bibliometric evaluation of formative research in the Faculty of Veterinary Medicine and Zootechnics of the Universidad Peruana Cayetano Heredia in the period 2012-2017

Thalía Llalla Vidal ${ }^{1}$, Galy Mendoza Torres ${ }^{2}$, Néstor Falcón Pérez ${ }^{2}$

\section{RESUMEN}

El objetivo del estudio fue evaluar las tesis desarrolladas en la Facultad de Medicina Veterinaria y Zootecnia de la Universidad Peruana Cayetano Heredia (FAVEZ-UPCH) durante el periodo 2012-2017. Para ello se diseñó un estudio observacional, descriptivo, retrospectivo, que utilizó herramientas bibliométricas para sistematizar la información de la base de datos del Banco de Tesis de la FAVEZ-UPCH. Se estudiaron las siguientes variables: año de sustentación, calificación de la tesis, línea de investigación, diseño de estudio, individuos u objeto de estudio, especie o muestra especifica evaluada, área o laboratorio del asesor principal, literatura utilizada, literatura actualizada y tesis transformada en artículos. La información obtenida se resumió mediante estadística descriptiva. Se evaluaron 195 tesis. El mayor número de tesis se sustentó durante el año 2014 (22,6\%). Las líneas de investigación priorizadas fueron Animales de Compañía y Animales de Producción (36,4\%), Salud Ambiental y Salud Pública (30,2\%) y Especies Productivas Nativas y Animales Silvestres (28,2\%), Los estudios observacionales fueron los más frecuentes $(90,3 \%)$, destacando los observacionales de corte transversal $(39,5 \%)$ y los descriptivos $(30,8 \%)$. Los animales domésticos fueron los más estudiados $(43,6 \%)$, seguidos de los silvestres $(30,3 \%)$, siendo los canes $(29,2 \%)$ y el hombre $(15,4 \%)$ las especies más estudiada. El Área de Epidemiología y Salud Pública $(30,7 \%)$ y la Clínica Veterinaria Docente Cayetano Heredia $(21,0 \%)$ desarrollaron la mayoría de tesis. Se utilizó 6548 referencias, predominando artículos científicos en idioma ingles $(32,1 \%)$ y español (20,4\%). Del total, solo el $29 \%$ fueron consideradas actualizadas. El 23,1\% de las tesis se convirtieron en artículos científicos y la mayoría se publicó en revistas nacionales $(91,2 \%)$. Los resultados permiten identificar áreas de la carrera que pueden ser estimuladas a desarrollar, mejorando la cobertura de la investigación formativa de la FAVEZ-UPCH en el campo de las competencias de las ciencias veterinarias.

PALABRA CLAVE: Bibliografía, investigación, tesis, veterinaria.

\section{SUMMARY}

The objective of the study was to evaluate thesis developed in the Faculty of Veterinary Medicine and Animal Science of Universidad Peruana Cayetano Heredia (FAVEZ- UPCH) during de period 2012-2017. For this mean an observational, descriptive and retrospective study was design; which used bibliometric tools to organize information from the database of FAVEZ-UPCH thesis bank. The variables studied were year of thesis presentation, qualification of the thesis, line of research, research design, object of study, specific species or sample evaluated, area or laboratory of principal advisor, type of used literature, actualized literature and number of thesis which developed 
in to scientific articles. Data obtained was summarized with descriptive statistics. 195 thesis were evaluated. the year with more thesis presentations was 2014 (22.6\%). Prioritized lines of research were companion animals and large animals (36.4\%), environmental health and public health $(30.2 \%)$ and native productive species and wild animals (28.2\%); observational studies were the most frequent (90.3\%) predominating the observational transversal cut studies (39.5\%) and descriptive ones (30.8\%). Domestic animals were the most studied (43.6\%), followed by wild animals (30.3\%), being dog (29.2\%) and men (15.4\%) the most studied species. Epidemiology and public health area (30.7\%) and Cayetano Heredia Veterinary Clinic (21.0\%) developed the greater number of thesis. 6548 references were used, predominating scientific articles in English (32.1\%) and Spanish (20.4\%). Of all just 29\% were considered actualized references. $23.1 \%$ of thesis were developed in scientific articles and the majority was published in national journals (91.2\%). The results of this study allow to identify areas on the faculty which can be developed hence improving the coverage of formative investigation in FAVEZ-UPCH on the veterinary science field.

KEY WORDS: bibliography, investigation, thesis, veterinary.

\section{INTRODUCCIÓN}

El producto de la investigación científica resulta en un nuevo conocimiento, este nuevo conocimiento adquiere valor cuando se publica y posteriormente, aplicado en el campo específico de la investigación, contribuye al desarrollo de la sociedad. La bibliometría juega un papel crucial, ya que le da valor medible al resultado de dicha actividad científica (Tricco, Runnels, Sampson y Bouchard, 1999). Esto permite establecer rankings según el nivel de producción de los autores u otros elementos obtenidos por agregación que se comparen (Ardanuy, 2012).

La bibliometría aplica las matemáticas y el método estadístico a la publicación de los resultados de la investigación científica buscando cuantificar la actividad científica de quienes la producen para analizar el curso de la comunicación escrita o literaria de carácter científico (Karolinska Institutet Bibliometrics Project Group, 2008; Camps, 2008).

La bibliometría sirve como apoyo para la toma de decisiones y dirección de la investigación, y designar mejor los recursos económicos con una base medible. También contribuye al buen prestigio del investigador, incentivando así el dinamismo de la investigación (Koskinen et al., 2008; Hood y Wilson, 2001; Thelwall, 2007). Al término de un análisis bibliométrico, sus resultados permiten comparar las diferentes situaciones de desarrollo científico entre regiones, autores, revistas, y medir objetivamente su crecimiento o retroceso (Rodríguez, 2009)

Los estudios bibliométricos realizados a nivel de las universidades en el Perú han buscado que valorar la participación estudiantil en los trabajos de investigación y las tendencias de estos; ejemplo de ello encontramos en los trabajos realizados en la Universidad Nacional Mayor de San Marcos, entre ellos los realizados por San Martin y Pacheco (2008) en la Facultad de Medicina Veterinaria, por Quintana (2006) quien evaluó las tesis de la Facultad de Psicología, y por Sogi, Perales, Anderson y Bravo (2002) realizado en la Unidad de Investigación de la Facultad de Medicina y el de Valle y Salvador (2009) quienes reportan un análisis bibliométrico de las tesis de pregrado de la Facultad de Medicina. Otras referencias de estudios bibliométricos lo encontramos en los trabajos de Ordinola et al., (2014) realizado en la Facultad de Odontología de la Universidad San Martin de Porras.

Un estudio intitulado "Estudio Bibliométrico de las Tesis de Pregrado en la Facultad de Veterinaria y Zootecnia de la Universidad Peruana Cayetano Heredia. Periodo 2006-2011" realizado por Yarleque (2012) había hecho una primera evaluación de la investigación formativa, cuyos resultados se hace necesario contrastar con lo que se ha realizado en el periodo 2012 - 2017 a fin de determinar su evolución.

Por ello, es necesario evaluar los productos de la investigación formativa documentos que en los últimos años se encuentran a disposición del público a través del repositorio de la Universidad Peruana Cayetano Heredia; a fin de conocer las tendencias de la investigación formativa (tesis) posterior a la evaluación realizada por Yarleque (2012); para ello se debe de utilizar la investigación bibliométrica como herramienta que permita obtener indicadores que valoren el estado de la investigación formativa que a 
su vez permitirá reformular, priorizar o potencializar las líneas de investigación que la Facultad de Medicina Veterinaria y Zootecnia ha definido desde el año 2015.

En ese contexto, el objetivo del estudio fue evaluar los trabajos de tesis desarrollados en la Facultad de Medicina Veterinaria y Zootecnia de la Universidad Peruana Cayetano Heredia durante el periodo 20122017.

\section{MATERIAL Y MÉTODOS}

El estudio se realizó en la Facultad de Medicina Veterinaria y Zootecnia de la Universidad Peruana Cayetano Heredia (FAVEZ - UPCH), teniendo como fuente de información la base de datos de proyectos de tesis y el Banco de Tesis de la Oficina de Promoción de la Titulación, oficina adjunta a la Escuela Profesional de Medicina Veterinaria y Zootecnia.

El estudio correspondió a una investigación observacional, descriptivo, retrospectivo, que utilizó herramientas bibliométricas para el análisis de las tesis de pregrado sustentadas en la FAVEZ-UPCH durante el periodo 2012-2017.

La recolección de datos se realizó mediante un instrumento elaborado para el estudio en donde se consideró las siguientes variables:

a. Año de sustentación de la tesis (entre 2012 y 2017)

b. Línea de investigación (definidas por la FAVEZ$\mathrm{UPCH}$ :

- Salud Ambiental y Salud Pública

- Animales de Compañía y Animales de Producción

- Especies Productivas Nativas y Animales Silvestres

- $\quad$ Especies Hidrobiológicas y Acuicultura

- Bienestar Animal y Modelos Animales para Investigación y Docencia

c. Diseño de estudio. - Se manejó el criterio utilizado por Yarleque (2012):

- Estudios experimentales.- En los que existe la manipulación del factor causal por parte del investigador para la determinación posterior del efecto.

- Estudios observacionales. - No interviene la mano del investigador para modificar ningún factor.

- Correlacionales. - Se miden dos o más variables, y se pretende establecer si éstas están relacionadas, y que grado de relación existe entre ellas.
- Corte Transversal. - En este estudio, las mediciones se hacen en una sola oportunidad. No existe período de seguimiento.

- Retrospectivo. - Basada en la revisión de datos extraídos de archivos, por ende, el inicio del estudio es posterior a los hechos estudiados.

- Descriptivo. - Describe las características de un objeto u animal sin buscar relaciones entre variables.

d. Individuos $u$ objeto de estudio:Animales domésticos

- Animales silvestres

- Hombre

- Cosas (Ej. Vacunas)

- Ambiente

e. Especie o muestra especifica de estudio. - Detalla la unidad de estudio específica.

f. Área o laboratorio a la que pertenecía el Asesor principal: Clínica Veterinaria Docente Cayetano Heredia

- Laboratorio de Anatomía

- Laboratorio de Biología Molecular

- Laboratorio de Epidemiología y Salud Pública

- Laboratorio de Microbiología

- Laboratorio de Nutrición

- Laboratorio de Parasitología

- Laboratorio de Patología Clínica

- Laboratorio de Reproducción

- $\quad$ Laboratorio de Vida Silvestre

- Unidad de Producción

- Literatura o bibliografía utilizada

- $\quad$ Artículo científico Ingles

- Artículo científico español

- Artículo científico en un tercer idioma

- Libro

- $\quad$ Página Web

- $\quad$ Tesis

- Leyes/normas

- Diarios/periódicos

- Otros (paginas libre, abstracto, documentos en PDF, Boletines informativos, revistas institucionales, comunicaciones).

g. Literatura actualizada: Se contabilizó la literatura utilizada los cinco años anteriores a la sustentación de la tesis

h. Tesis convertidas en artículos: Información obtenida a partir de la búsqueda en Google Académico.

Se utilizó la totalidad de trabajos de investigación bajo la denominación de tesis. Para la obtención de la información se realizó la lectura de los resúmenes, 
metodología y literatura citada de las tesis con la finalidad de recolectar la información necesaria. Cuando fue necesario se realizó la lectura de la tesis en su totalidad.

La información obtenida a partir de la revisión de los proyectos de tesis y tesis fue transferida a una base de datos en el programa Microsoft Excel. Los datos se ingresaron utilizando un lenguaje numérico con la finalidad de facilitar los cálculos estadísticos.
El procesamiento de datos se realizó utilizando el programa STATA 13.0. Los datos fueron resumidos en cuadros o figuras donde se presentaron las frecuencias absolutas y relativas obtenidas.

El proyecto fue aprobado por el comité institucional de ética UPCH mediante el dictamen Nro. CAREGORVEI-031-018.

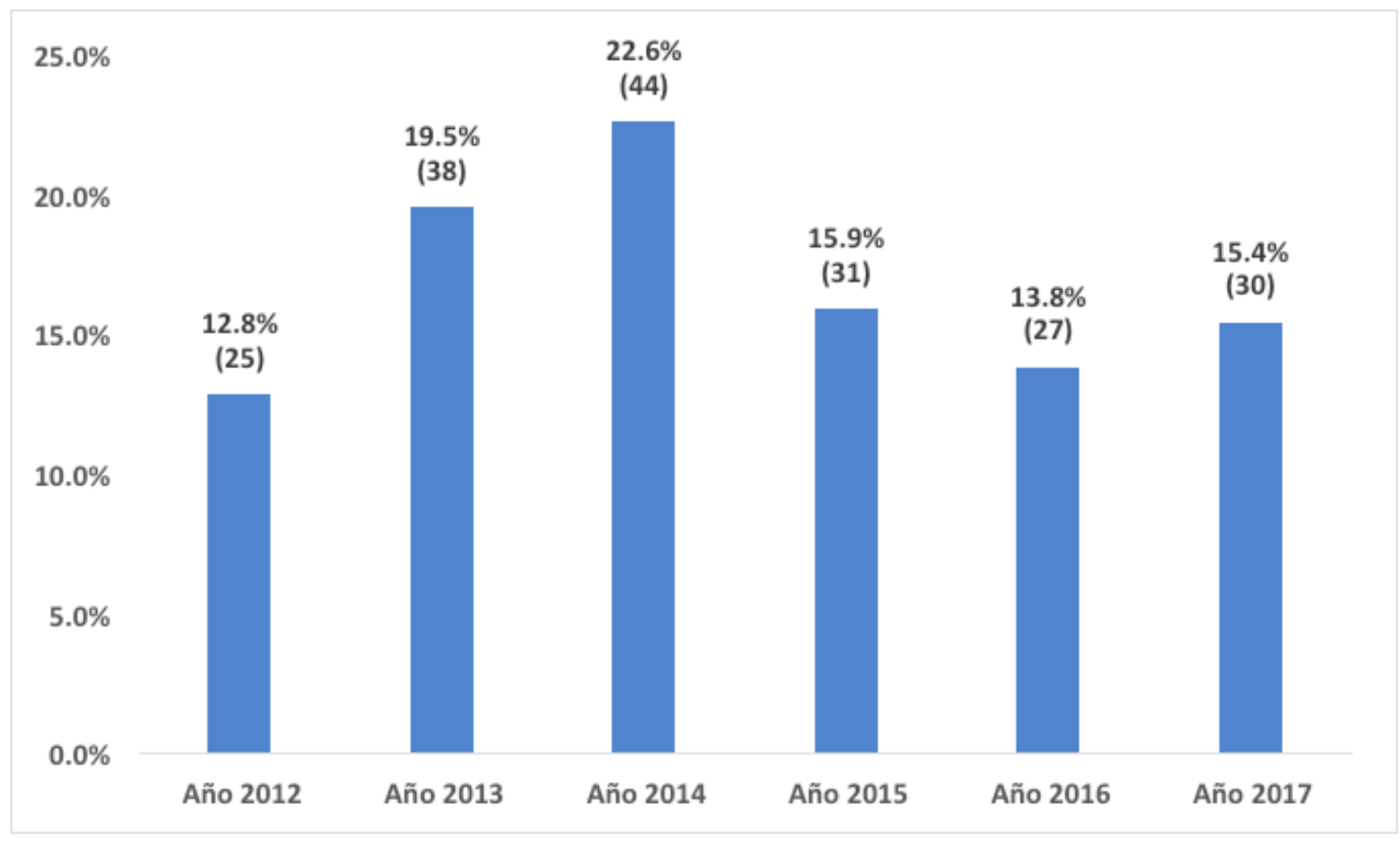

Figura 1. Distribución de las tesis de acuerdo al año de sustentación. Facultad de Medicina Veterinaria y Zootecnia - Universidad Peruana Cayetano Heredia. Periodo $2012-2017$ (n=195).

Tabla 1. Clasificación de las tesis de acuerdo con la línea de investigación y tipo de estudio. Facultad de Medicina Veterinaria y Zootecnia - Universidad Peruana Cayetano Heredia. Periodo 2012 - 2017 $(n=195)$.

\begin{tabular}{clcc}
\hline Variable & \multicolumn{1}{c}{ Estrato de la variable } & $\mathrm{n}$ & $\%$ \\
\hline & Animales de Compañía y Animales de Producción & 71 & 36,4 \\
& Salud Ambiental y Salud Pública & 59 & 30,2 \\
Líneas de & Especies Productivas Nativas y Animales Silvestres & 55 & 28,2 \\
Investigación & Especies Hidrobiológicas y Acuicultura & 5 & 2,6 \\
& Bienestar Animal y Modelos Animales para & 5 & 2,6 \\
& Investigación y Docencia & 77 & 39,5 \\
& Observacional corte transversal & 60 & 30,8 \\
& Observacional descriptivo & 34 & 17,4 \\
& Observacional Retrospectivo & 19 & 9,7 \\
& Experimentales de estudio & 5 & 2,6 \\
\hline
\end{tabular}


Tabla 2. Clasificación de las tesis de acuerdo con la categoría del individuo de estudio y la especie específica. Facultad de Medicina Veterinaria y Zootecnia - Universidad Peruana Cayetano Heredia. Periodo $2012-2017$ ( $\mathrm{n}=195)$.

\begin{tabular}{llcc}
\hline \multicolumn{1}{c}{ Variable } & \multicolumn{1}{c}{ Estrato de la variable } & $\mathrm{n}$ & $\%$ \\
\hline \multirow{3}{*}{ Categoría del } & Animal domestico & 85 & 43,6 \\
individuo de estudio & Animal silvestre & 59 & 30,3 \\
& Hombre & 33 & 16,9 \\
& Cosa & 12 & 6,2 \\
& Ambiente & 5 & 2,6 \\
& Perro & 57 & 29,2 \\
Hombre & 30 & 15,4 \\
Especie/muestra & Cuy & 14 & 7,2 \\
Cspecifica & Bomélidos sudamericanos & 11 & 5,6 \\
& Primates & 11 & 5,6 \\
& Aves de corral & 6 & 3,1 \\
& Ranas & 6 & 3,1 \\
Paloma & 5 & 2,6 \\
& Perro y gato & 5 & 2,6 \\
Gato & 4 & 2,1 \\
& Agua & 4 & 2,1 \\
Cerdo & 4 & 2,1 \\
& Otros* & 3 & 1,5 \\
\hline
\end{tabular}

*Harina de pescado (2), majaz (2), rata (2), tesis (2), tortuga (2), agua / molusco bivalvo (1), alevinos de gamitana(1), alimento (1), animales de producción (1), bovino/alpaca (1), caballo de paso (1), carne (1), carnívoros silvestres (1), cocodrilo (1), fauna silvestre y productos derivados (1), galpón (1), gaviota (1), heces (1), lactobacillus sp, leche (1), manatí (1), margay (1), murciélago/animales de traspatio (1), nutria (1), ordenanzas municipales (1), peces (1), queso (1), suelo (1), tamandúa (1), ulomoides (1), varias especies (1)

\section{RESULTADOS}

En el periodo 2012 al 2017 se sustentaron 195 tesis, siendo el número de sustentaciones mayor el año 2014 (22.6\%). La distribución de las tesis según año de sustentación se presenta en el gráfico 1 . Del total de tesis sustentadas, el $46.2 \%$ (90) se realizaron en la capital, el 30.8\% (60) fueron realizadas en provincias y el 23.1\% (45) se realizó en la FAVEZ-UPCH.

La distribución de las tesis según línea de investigación encontró que predominaban las de Animales de Compañía y Animales de Producción $(36,4 \%)$, seguidas por las de Salud Ambiental y Salud Pública (30,2\%), y Especies Productivas Nativas y Animales Silvestres (28,2\%). El diseño de estudio que se planteó con mayor frecuencia correspondió a los observacionales alcanzando el 90,3\% (tabla 1).

La clasificación de los estudios según el individuo u objeto de estudio reportó que los animales domésticos fueron los más empleados en los trabajos de investigación (43,6\%), seguidos por los animales silvestres $(30,3 \%)$. En relación con la especie estudiada o la muestra específica se encontró que los canes $(29,2 \%)$ y el hombre $(15,4 \%)$ fueron los más estudiados (tabla 2).

La distribución de las tesis sustentadas de acuerdo con el área o laboratorio a la que pertenecía el asesor principal muestra que predomina el Área de Epidemiología y Salud Pública $(30,7 \%)$ y la Clínica Veterinaria Docente Cayetano Heredia $(21,0 \%)$ (tabla 3$)$.

El volumen de la literatura citada alcanzó un total de 6548 referencias. Los más comunes fueron los artículos científicos los que se incluyen aquellos redactados en idioma ingles (32.1\%) y español (20.4\%) (tabla 4). Del total de referencias utilizadas, $1883(29 \%)$ correspondieron a referencias publicadas dentro de los 5 años anteriores a la sustentación de la tesis, fueron consideradas como actualizadas. 
Tabla 3. Distribución de las tesis de acuerdo al área o Laboratorio del asesor principal. Facultad de Medicina Veterinaria y Zootecnia - Universidad Peruana Cayetano Heredia. Periodo $2012-2017(\mathrm{n}=195)$.

\begin{tabular}{|c|c|c|}
\hline Área o laboratorio & $\mathrm{n}$ & $\%$ \\
\hline Epidemiología y Salud Pública & 60 & 30,7 \\
\hline Clínica Veterinaria Docente Cayetano Heredia & 41 & 21 \\
\hline Laboratorio de Parasitología & 16 & 8,2 \\
\hline Laboratorio de Microbiología & 14 & 7,2 \\
\hline Laboratorio de Vida Silvestre & 14 & 7,2 \\
\hline Laboratorio de Anatomía & 13 & 6,7 \\
\hline Laboratorio de Biología Molecular & 10 & 5,1 \\
\hline Unidad de Producción & 10 & 5,1 \\
\hline Laboratorio de Reproducción & 8 & 4,1 \\
\hline Laboratorio de Patología Clínica & 5 & 2,6 \\
\hline Laboratorio de Nutrición & 4 & 2,1 \\
\hline Total & 195 & 100 \\
\hline
\end{tabular}

Tabla 4. Distribución del tipo de literatura utilizada en las tesis. Facultad de Medicina Veterinaria y Zootecnia - Universidad Peruana Cayetano Heredia. Periodo 2012 - 2017 ( $\mathrm{n}=195)$.

\begin{tabular}{lcc}
\hline \multicolumn{1}{c}{ Tipo de literatura } & $\mathrm{n}$ & $\%$ \\
\hline Artículo científico Ingles & 2112 & 32,1 \\
Artículo científico español & 1344 & 20,4 \\
Libro & 1128 & 17,1 \\
Tesis & 515 & 7,8 \\
Página Web & 468 & 7,1 \\
Artículo científico otro idioma & 63 & 1 \\
Leyes/normas & 67 & 1 \\
Diarios/periódicos & 33 & 0,5 \\
Otros* & 854 & 13 \\
& 6584 & 100 \\
\hline
\end{tabular}

*paginas libre, abstracto, documentos en PDF, Boletines informativos, revistas institucionales, comunicaciones.

Tabla 5. Distribución de los artículos según revista en la que se publicaron las tesis. Facultad de Medicina Veterinaria y Zootecnia - Universidad Peruana Cayetano Heredia. Periodo 2012 - 2017 ( $\mathrm{n}=45$ ).

\begin{tabular}{lccc}
\multicolumn{1}{c}{ Revista } & $\mathrm{n}$ & $\%$ & Indización \\
\hline Salud y Tecnología Veterinaria & 25 & 55,6 & Latindex \\
Revista de Investigaciones Veterinarias del Perú & 13 & 28,9 & Scopus \\
Revista de Ciencias Veterinarias & 2 & 4,4 & No index \\
Journal of wildlife diseases & 1 & 2,2 & Scopus \\
Revista do Instituto de Medicina Tropical de São Paulo & 1 & 2,2 & Scopus \\
Revista Panamericana de Salud Pública & 1 & 2,2 & Scopus \\
Revista Peruana de Medicina Experimental y Salud Publica & 1 & 2,2 & Scopus \\
Chelonian Conservation and Biology, & 1 & 2,2 & Scielo \\
\hline
\end{tabular}


El número de tesis que se convirtieron en artículos científicos alcanzó a 45 lo que representa el 23,1\% del total. La mayor cantidad se publicaron en la revista Salud y Tecnología Veterinaria $(55,6 \%$ ) y la Revista de Investigaciones Veterinarias del Perú (28,9\%) (tabla $5)$.

\section{DISCUSIÓN}

La tesis es un trabajo de investigación que por definición debe de realizar algún aporte al conocimiento existente y poner de manifiesto la capacidad del estudiante para el trabajo intelectual serio y sistemático, el cual por más limitada que sea en sus alcances, tiene que demostrar los conocimientos y la pericia metodológica de su autor (Arias, 2006). Este tipo de trabajo ha sido el medio para alcanzar la titulación de 195 egresados de la FAVEZ-UPCH en el periodo 2012 - 2017, los cuales forman parte del estudio bibliométrico que se presenta.

No se encontró una tendencia al aumento en el número de tesis sustentadas conforme pasaban los años, tal como si encontró Yarleque (2012) en su investigación, quien encontró que el número de tesis sustentadas se fue incrementando conforme avanzaban los años.

El número de tesis sustentadas por año debería de estar estimulado por la necesidad que deben de tener los egresados de contar con el título profesional a fin de ejercer en forma legal la carrera profesional. La práctica clínica con animales de compañía es una de las áreas en donde la posesión del título profesional es crítica. En ella se debe de prescribir tratamientos o firmar certificados, por lo que la ausencia del título profesional resulta en una falta al Código Deontológico del Colegio Médico Veterinario del Perú y es susceptible de ser denunciado antes las autoridades del ministerio público por ejercicio ilegal de la profesión.

En cuanto a la ubicación geográfica mencionada para el desarrollo de la tesis, los resultados muestran que la mayoría de los trabajos se realizaron en Lima, sin embargo, esta proporción ha disminuido en comparación a los hallazgos de Yarlequé (2012) quien en un estudio similar para el periodo 2006 - 2011 en la FAVEZ-UPCH, encontró que el $84.5 \%$ de los trabajos de tesis se realizaron en el departamento de Lima.

Aun cuando no se encuentra documentado, la disminución de la brecha Lima - provincias, relacionado al lugar de ejecución de la tesis, ello estaría relacionado a las siguientes razones: a). obtención de facilidades para el desarrollo de la investigación cuando los estudiantes estuvieron realizando sus rotaciones en el interior del país, b). cuando los egresados que provienen de ella, decidieron realizar sus estudios en los lugares de donde proceden, y c). cuando los temas de investigación que provinieron de proyectos de los docentes que tuvieron como lugar de recolección de muestras o información el interior del país. También es importante destacar el aumento de las tesis que tienen como lugar de obtención de información la FAVEZ$\mathrm{UPCH}$, en donde la Clínica Veterinaria Docente Cayetano Heredia es la principal sede para ello.

En general, se estima que el desarrollo de la producción científica suele ser mayor cercano a la capital de los estados debido a una mayor oportunidad de desarrollo y facilidades para con ella. Así BravoVinaja y Sanz-Casado (2008) encontraron que en conjunto, más de $50 \%$ de la producción científica en ciencias agropecuarias en México se concentró en el Distrito Federal y el Estado de México (30,77\% y $22,71 \%$, respectivamente), que es la zona donde tienen su sede muchos centros de investigación en ciencias agrícolas.

El año 2015 la FAVEZ-UPCH definió cinco líneas de investigación: Animales de Compañía y Animales de Producción; Salud Ambiental y Salud Pública; Especies Productivas Nativas y Animales Silvestres; Especies Hidrobiológicas y Acuicultura; y Bienestar Animal y Modelos Animales para Investigación y Docencia. De ellas predominaron las tres primeras en orden descendente, albergando alrededor del 95\% de las tesis desarrolladas en el periodo de estudio. Ello representa un reto a fin de que las líneas de investigación menos desarrolladas sean incentivadas, dado que la FAVEZ-UPCH, a través de sus propios docentes ha juzgado que son importantes.

La evaluación de los diseños de estudio muestra que predominan los estudios observacionales y entre ellos los descriptivos y de corte transversal. Estos estudios se limitan a recolectar o medir variables, por lo que resultan ser más prácticos y factibles de realizar. Yarlequé (2012) menciona que entre los factores que favorecen la realización de este tipo de estudio está el poco tiempo y costo que involucra obtener la información, debido a que no hay necesidad de hacer seguimiento de individuos.

En contraste, la cantidad de estudios experimentales es exigua, lo que puede deberse al alto costo de inversión y dinero que se debe de hacer para desarrollar 
un estudio experimental de calidad. Hernandez, Fernandez y Baptista (2010) definen los experimentos como estudios de intervención donde el investigador genera situaciones manipulando una o más variables para ver su efecto en quienes participan del estudio en comparación a un control, bajo condiciones controladas por el mismo. Cumplir estas condiciones hace que los estudios experimentales sean los menos requeridos por los egresados que buscan titularse a la brevedad posible una vez que culminan sus estudios.

En concordancia con la línea de investigación que predomina en los trabajos de tesis, lo individuos involucrados en los estudios correspondieron a los animales domésticos en mayor proporción. De ellos, lo canes fue la especie más estudiada. Esto resultados son similares a los obtenidos por Yarleque (2012) en la FAVEZ-UPCH (periodo 2006 - 2011) y los obtenidos por San Martín y Pacheco(2008) en la Facultad de Medicina Veterinaria de la Universidad Nacional Mayor de San Marcos (FMV-UNMSM) (periodo 2001-2006). En ambos casos se argumenta que esta preferencia estaría relacionada a que los egresados en su mayoría tienen la predilección por desarrollarse profesionalmente en el campo de la clínica de animales de compañía, a lo que se suma la facilidad para realizar investigaciones con estos animales por ser los más difundidos, tener acceso a una Clínica Veterinaria Universitaria y la disposición de los docentes del área para asesorar los trabajos de investigación.

Después de los canes, el hombre resulta ser la especia más estudiada. Los trabajos de investigación en esta área buscan información acerca de las consecuencias de las enfermedades zoonóticas, la identificación de conocimientos y prácticas riesgosas en relación humano-animal y en los estudios de estimación de canes con dueño, en donde las personas responden las preguntas de las encuestas desarrolladas para ese fin. Yarlequé (2016) menciona que el buscar información en las personas permite visualizar las consecuencias que producen en el hombre descuidos en las medidas de prevención de las zoonosis, así como recoger información de conocimientos, prácticas y tendencias, que suele ser de utilidad para los profesionales que laboran en el área de la salud pública. A ello se debe de sumar el aporte de los estudios de estimación de canes los que deberían de servir para que las autoridades del Ministerio de Salud y municipales distritales, programen adecuadamente los requerimientos para las campañas de vacunación antirrábica (VANCAN) y los programas de tenencia responsable de animales de compañía (TRAC) respectivamente.
Los estudios en camélidos sudamericanos y cobayos requieren especial interés. Santos (2009) y Morales (2011) indican que estas especies son las que tienen mayor proyección como alternativa productiva para el desarrollo de las economías regionales. Otra área que aparece como promisoria es la de acuicultura, aunque aún no se destaca en los resultados, el trabajo directo con peces e hidrobiológicos, la atención del medio en los que se desarrolla su crianza ha sido motivo de investigación en el periodo de estudio. Las otras especies se quedan relegadas como objetos de estudio, por lo que se hace necesario se estimule la investigación en ellas y las líneas de investigación que las involucran.

En un estudio similar realizado por San Martin y Pacheco (2008) encontraron que las especies tradicionales de investigación en las tesis de pregrado de la FMV-UNMSM fueron los bovinos y los caninos; seguido de las cuatro especies de camélidos sudamericanos. Ello puede estar relacionado a que dicha institución posee, además de una Clínica Veterinaria para animales de compañía en Lima, sedes en el interior del país en donde se desarrollan investigaciones en las especies bovinas (IVITA - Mantaro) y camélidos sudamericanos (IVITA - La Raya).

En cuanto al área o laboratorio a los que se encuentran vinculados los asesores principales de las tesis, se destaca claramente el área de Epidemiología y Salud Pública y la Clínica Veterinaria Docente Cayetano Heredia, siguiendo el patrón de las especies específicas que han sido involucradas en los estudios de tesis. En el primer caso tiene importancia el desarrollo del área considerando que la medicina veterinaria forma parte de las profesiones de la salud, cuya misión es proporcionar a la población las condiciones necesarias para desarrollar una vida saludable. En el caso del segundo, destaca su desarrollo debido probablemente a que representa el área de predilección de los futuros profesionales egresados de la FAVEZ-UPCH y donde los temas clínicos y de salud animal toman especial interés. San Martín y Pacheco (2008) señalan que en la FMV-UNMSM, el área que más tesis ha producido fue la de salud animal con el $75 \%$, seguido por producción animal con $17 \%$. Ello considera que esto estaría asociado al mayor número de docentes investigadores en esta área y, el reforzamiento, en los últimos años, de los aspectos médicos de la profesión a expensas del área de producción animal.

La clasificación de las tesis de acuerdo al laboratorio de procedencia del asesor principal también lo realiza 
San Martin y Pacheco (2008) en la FMV-UNMSM. Ellos encuentran que el Laboratorio de Microbiología y Parasitología Veterinaria tuvo la responsabilidad del mayor número de tesis (37\%), seguido por el de Patología Aviar con 12\%, Medicina Preventiva con $8.5 \%$ y Reproducción y Obstetricia Veterinaria con 7.7\%. Asimismo, las clínicas de Animales Mayores y Menores en conjunto produjeron el $8.5 \%$ de las tesis.

Es importante estimular la investigación formativa en las diferentes áreas en las que se desarrollan los Médicos Veterinarios, considerando, además, que todas ellas presentan relevancia dentro de la curricula de la carrera en la FAVEZ-UPCH. Yarlequé (2016) había considerado que las áreas de fauna silvestre, producción, laboratorios y los estudios económicos tenían un futuro promisorio como áreas para el desarrollo de trabajos de investigación.

El tipo de literatura que predomina en el estudio fueron los artículos científicos, tanto los escritos en idioma ingles como español. Si bien es cierto que en proporción son más que otras fuentes, ello ha tenido un retroceso, especialmente en el uso de la literatura en idioma extranjero, en relación a los encontrado por Yarleque (2012), los que alcanzaron a 42,9\% y 15,8\%, en contraste con el presente estudio en donde se alcanza $32,1 \%$ y $20,4 \%$, para los artículos en inglés y español respectivamente

En este aspecto, Huamaní y Pacheco (2012) mencionan que toda investigación debe partir de una adecuada búsqueda de la literatura y es el autor quien seleccionará aquellas referencias que justificadamente aportan a su investigación. Arango (2010) señala que el uso correcto de las citas y la inclusión de las referencias bibliográficas demuestran que la persona se ha documentado adecuadamente y como consecuencia logra resaltar las aportaciones de otros estudios. Por ello, se debe de estimular la búsqueda, lectura, revisión y críticas de artículos en revistas científicas indexadas y en idioma extranjero a fin de exponer a los estudiantes y egresados en contacto con la información más actualizada posible.

La proporción de tesis que fueron llevadas a artículos científicos alcanzó una proporción mayor que los reportados por Yarleque (2012) para el periodo 2006 - 2011 (22,1\% versus 15,5\%). Esta mejora posiblemente estaría asociada a la aparición de la Revista Salud y Tecnología Veterinaria, que es el medio de difusión oficial de la FAVEZ-UPCH. Esta revista tiene una circulación semestral y publica artículos de investigación originales relacionados con el campo de la medicina veterinaria, zootecnia, salud pública veterinaria, fauna silvestre, ciencias básicas afines, y tecnología aplicada a la investigación, bienestar, manejo, crianza, producción y salud animal. A esta revista se dirigió más de la mitad de los trabajos publicados, seguidos por los publicados en la Revista de Investigaciones Veterinarias del Perú. En cuanto al idioma de publicación de los artículos, predominó el español, ello estaría asociado al país de origen de las principales revistas a las que están dirigidas las investigaciones.

Es importante la publicación en revistas científicas debido a que ellas son vehículos de transmisión del conocimiento, son recursos esenciales para el aprendizaje, son medios de comunicación de los nuevos hallazgos obtenidos gracias a la investigación, y además, juegan un papel trascendental en la divulgación de las ciencias y sus avances, y en la promoción del desarrollo científico (Blanco, 2011). La elección de la revista también es importante debido a que entre más internacional sea la revista y sus contenidos, mayor visibilidad tendrán sus contenidos y sus autores (López, 2010).

Si bien es cierto que por un lado se alcanzó una mayor proporción de publicaciones, por otro lado, se disminuyó la proporción de artículos publicados en revistas que se encuentran en bases de indización más elevadas que la que esta indizada la revista de la FAVEZ-UPCH. En ese sentido se hace necesario sensibilizar a tesistas y asesores para que los trabajos de tesis de alta calidad, se conviertan en artículos científicos y sean enviados a publicación a revistas extranjeras o revistas nacionales con altos niveles de indización lo que permitirá que el conocimiento obtenido alcance una mayor difusión. Ello no quiere decir que se descuide la publicación en la revista Salud y Tecnología Veterinaria a la que se pueden dirigir aquellos estudios cuyo conocimiento obtenido tiene interés local o regional, así como también ella puede albergar a artículos científicos provenientes de otras instituciones lo que permitiría cumplir con requisitos para lograr el acceso a bases de datos de mayor difusión.

Los resultados del estudio deben servir como diagnóstico del comportamiento de la investigación formativa a fin de identificaráreas de la carrera de medicina veterinaria que deben ser incentivadas a desarrollarse. Esto se puede hacer a través de la generación de temas de investigación para estudios de tesis que pueden ser elegidos por los egresados de la FAVEZ-UPCH, los que a ser culminados y en lo posible publicados en una revista de investigación, permitirán visualizar las 
actividades que los docentes y laboratorios de la FAVEZ$\mathrm{UPCH}$, contribuyendo a su posicionamiento dentro de las instituciones educativas de formación profesional en medicina veterinaria en el Perú.

\section{CONCLUSIONES}

El estudio de investigación bilbiométrica de las tesis sustentadas en la FAVEZ-UPCH durante el periodo 2012-2017, llega a las siguientes conclusiones:

Durante el periodo de estudio se sustentaron 195 con una distribución según línea de investigación de: Animales de Compañía y Animales de Producción (36,4\%), Salud Ambiental y Salud Pública (30,2\%) y Especies Productivas Nativas y Animales Silvestres $(28,2 \%)$.

Losestudiosobservacionales fueronlosmás frecuentes $(90,3 \%)$, predominando los tipos observacionales de corte transversal (39,5\%) y los descriptivos $(30,8 \%)$.

Los animales domésticos fueron los más estudiados $(43,6 \%)$, seguidos de los silvestres $(30,3 \%)$, siendo los canes $(29,2 \%)$ y el hombre $(15,4 \%)$ las especies especificas más estudiadas.

El Área de Epidemiología y Salud Pública (30,7\%) y la Clínica Veterinaria Docente Cayetano Heredia $(21,0 \%)$ desarrollaron la mayoría de las tesis sustentadas en el periodo de estudio.

Se utilizó un total de 6548 referencias bibliográficas en las 195 tesis sustentadas, predominando artículos científicos en idioma ingles $(32,1 \%)$ y español $(20,4 \%)$, de los cuales solamente $29 \%$ fueron consideradas actualizadas (publicada 5 años atrás de la fecha de sustentación de la tesis).

El $22.1 \%$ de las tesis se convirtieron en artículos científicos y la mayoría se publicó en revistas nacionales $(92,8 \%)$, siendo la revista Salud y Tecnología Veterinaria $(55,6 \%)$ y la Revista de Investigaciones Veterinarias del Perú $(28,9 \%)$ los destinatarios.

\section{Correspondencia:}

Néstor Falcón Pérez

Correo electrónico:

nestor.falcon@upch.pe

\section{REFERENCIAS BIBLIOGRAFIA}

1. Ardanuy, J (2012). Breve introducción a la bibliometría. Barcelona: Universitat de Barcelona. Karolinska Institutet Bibliometrics Project Group (2008). Bibliometrics. Publication Analysis as a Tool for Science Mapping and Research Assessment. Stockholm: Karolinska Institutet Bibliometrics Project Group.

2. Blanco, F. S. (2010). Análisis bibliométrico de la revista" Educación" de la Pontificia Universidad Católica del Perú (1992-2005). Alexandria, 4(7), 13-27.

3. Bravo-Vinaja, Á., \& Sanz-Casado, E. (2008). Análisis bibliométrico de la producción científica de México en Ciencias Agrícolas durante el periodo 1983-2002. Revista fitotecnia mexicana, 31(3):00.

4. Camps, D. (2008). Limitaciones de los indicadores bibliométricos en la evaluación de la actividad científica biomédica. Colombia Médica, 39(1), 7479.

5. Hernandez, R., Fernandez, C., \& Baptista, M. (2010). Metodología de la investigación. 5ta ed. Ciudad de México: McGraw-Hill, 121-122.

6. Hood, W., \& Wilson, C. (2001). The literature of bibliometrics, scientometrics, and informetrics. Scientometrics, 52(2), 291-314.

7. Koskinen, J., Isohanni, M., Paajala, H., Jääskeläinen, E., Nieminen, P., Koponen, H., ... \& Miettunen, J. (2008). How to use bibliometric methods in evaluation of scientific research? An example from Finnish schizophrenia research. Nordic journal of psychiatry, 62(2), 136-143.

8. López, W. L. (2010). Internacionalización y visibilidad del conocimiento. Universitas Psychologica, 9(2), 311-314.

9. Ordinola, S. C., Tello, Ch. V., Vargas, P. J., Rivera, V. R., \& Alfaro, C. D. (2014). Análisis de las tesis de pregrado de la Facultad de Odontología de una universidad peruana, 2005-2013. KIRU,11(1),2531

10. Quintana P. A. (2006). Análisis neo-bibliométrico de las Investigaciones de tesis en la Escuela Académico-Profesional de Psicología UNMSM. Revista de investigación en psicología, 9(1), 8199.

11. Rodríguez, M. D., Sáenz, R. G., Arroyo, H. M., Herrera, D. P., De la Rosa Barranco, D., \& 
Caballero-Uribe, C. V. (2009). Bibliometría: conceptos y utilidades para el estudio médico y la formación profesional. Salud Uninorte, 25(2), 319330 .

12.San Martín, H., \& Pacheco, M. (2008). Análisis bibliométrico de las tesis de pregrado de la Facultad de Medicina Veterinaria de la Universidad Nacional Mayor de San Marcos en el periodo 2001-2006. Revista de Investigaciones Veterinarias del Perú, 19(1), 82-92.

13. Sogi, C., Perales, A., Anderson, A., \& Bravo, E. (2002). Producción científica de los investigadores de la Facultad de Medicina, UNMSM. Tendencia 1991-2000. Anales de la Facultad de Medicina, 63(3),191-200. Thelwall, M. (2008). Bibliometrics to webometrics. Journal of information science, 34(4), 605-621.
14.Tricco, A. C., Runnels, V., Sampson, M., \& Bouchard, L. (2008). Shifts in the use of population health, health promotion, and public health: a bibliometric analysis. Can J Public Health, 99(6),466-71.Valle, R., \& Salvador, E. (2009). Análisis bibliométrico de las tesis de pregrado de la Facultad de Medicina de la Universidad Nacional Mayor de San Marcos. Anales de la Facultad de Medicina, 70(1): 11-18.

15. Yarlequé C. (2012). Estudio Bibliométrico de las Tesis de Pregrado en la Facultad de Veterinaria y Zootecnia de la Universidad Peruana Cayetano Heredia. Periodo 2006-2011. Tesis de Pregrado. Facultad de Medicina Veterinaria y Zootecnia. Universidad Peruana Cayetano Heredia. Lima, Perú. 29p. 